\title{
A total of 404 cases of aortic valve reconstruction with glutaraldehyde-treated autologous pericardium
}

\author{
Shigeyuki Ozaki, MD, PhD, Isamu Kawase, MD, Hiromasa Yamashita, MD, Shin Uchida, MD, \\ Yukinari Nozawa, MD, Mikio Takatoh, MD, and So Hagiwara, MD
}

Objective: To determine the feasibility of original aortic valve reconstruction, 404 consecutive cases were reviewed. The early results are reported here.

\begin{abstract}
Methods: Aortic valve reconstruction was performed for 404 patients from April 2007 through September 2011. The results for all 404 patients were reviewed retrospectively. There were 289 patients with aortic stenosis and 115 patients with aortic regurgitation. One hundred two patients had bicuspid aortic valves, 13 patients had unicuspid valves, and 2 patients had quadricuspid valves. There were 201 males and 203 females. Mean age was $69.0 \pm 12.9$ years. Preoperative echocardiography revealed an average peak pressure gradient of $79.6 \pm 32.5$ $\mathrm{mm} \mathrm{Hg}$ with aortic stenosis. Surgical annular diameter was $20.3 \pm 3.2 \mathrm{~mm}$. The surgical procedure is based on the independent tricuspid replacement by autologous pericardium. First, the distance between the commissure is measured with an original sizing apparatus, then the pericardial cusp is trimmed using an original template, and it is sutured to the annulus.
\end{abstract}

Results: There were no conversions to prosthetic valve replacement. There were 7 in-hospital mortalities resulting from a noncardiac cause. Postoperative echocardiography revealed an average peak pressure gradient of $19.8 \pm 10.2 \mathrm{~mm} \mathrm{Hg} 1$ week after surgery and $13.8 \pm 3.7 \mathrm{~mm} \mathrm{Hg} 3.5$ years after surgery. Two patients needed reoperation because of infective endocarditis. The other 402 patients showed less than mild aortic regurgitation. No thromboembolic events were recorded. The mean follow-up period was $23.7 \pm 13.1$ months. Freedom from reoperation was $96.2 \%$ at 53 months of follow-up.

Conclusions: Original aortic valve reconstruction was feasible in patients with various aortic valve diseases. Long-term data will be disclosed in the future. (J Thorac Cardiovasc Surg 2014;147:301-6)

Atrioventricular valve repair has become more popular than valve replacement with its standardization and reproducibility. For aortic valve disease, bioprosthetic valves are being used increasingly for replacement because of the problems with anticoagulation of mechanical valves. However, despite the progress of the design and construction of prosthetic valves, hemodynamic performance is not yet comparable with that of native aortic valves. ${ }^{1}$ In recent years, much attention has been given to repairing aortic valve disease, with consequent improvement in postoperative results.

Many reports of aortic valve repair are limited to the treatment for aortic regurgitation (AR)., ${ }^{2,3}$ Our new style of aortic valve reconstruction can be applied to a wide spectrum of aortic valve diseases, including aortic stenosis (AS), AR, infective endocarditis (IE), prosthetic valve endocarditis (PVE), and annulo-aortic ectasia.

\footnotetext{
From the Department of Cardiovascular Surgery, Toho University Ohashi Medical Center, Tokyo, Japan.

Disclosures: Authors have nothing to disclose with regard to commercial support. Received for publication Aug 18, 2012; revisions received Oct 4, 2012; accepted for publication Nov 6, 2012; available ahead of print Dec 10, 2012.

Address for reprints: Shigeyuki Ozaki, MD, PhD, Department of Cardiovascular Surgery, Toho University Ohashi Medical Center, 2-17-6 Ohashi, Meguro, Tokyo, 153-8515 Japan (E-mail: ozakis@ oha.toho-u.ac.jp). $0022-5223 / \$ 36.00$

Copyright (c) 2014 by The American Association for Thoracic Surgery http://dx.doi.org/10.1016/j.jtcvs.2012.11.012
}

We basically replace all 3 aortic valve cusps by glutaraldehyde-treated autologous pericardium. We assume this technique is more like valve reconstruction than replacement because there is no use of foreign material and no need for postoperative anticoagulation. The main reason why we call this technique a reconstruction is that we can make new cusps, one by one, from the distance between each commissure in the operative field. There have been some reports of aortic valve reconstruction with autologous pericardium or stentless autologous pericardial aortic valve replacement. ${ }^{4,5}$ These reports mentioned the advantage of direct suturing of the pericardium to the annulus. ${ }^{6-9}$ With this method, surgeons can preserve the natural aortic root expansion in the systole with maximal effective orifice area, ${ }^{10-12}$ but they still replace the aortic valve as 1 whole structure. The difference in our original aortic valve reconstruction from others is the independent replacement of 3 cusps by 3 native-size autologous pericardial cusps. We consider the size of the aortic cusp to be defined by the distance between the commissures. In addition, aortic valves may represent a collection of different-size cusps. We believe independent replacement of cusps is more effective in preserving the natural motion of the aortic valve annulus and the coordination of the left ventricle, aortic annulus, sinus of Valsalva, and aorta. 


\section{Abbreviations and Acronyms \\ $\mathrm{AR}=$ aortic regurgitation \\ AS = aortic stenosis \\ IE = infective endocarditis \\ $\mathrm{PVE}=$ prosthetic valve endocarditis}

To inspect the feasibility of original aortic valve reconstruction, 404 consecutive patient results were reviewed.

\section{METHODS}

Our new original aortic valve reconstruction and the clinical study of this procedure were approved by the institutional review board of Toho University Ohashi Medical Center. All patients underwent this operation after written informed consent was obtained.

Our original aortic valve reconstruction was performed on 404 patients from April 2007 through September 2011. We reviewed retrospectively all 404 patients and evaluated short-term and mid-term results.

The indication of our original aortic valve reconstruction was first by echocardiography for the patients with a small aortic annulus with a diameter $\leq 21.5 \mathrm{~mm}$, and second for patients who rejected mechanical valve implantation. Among them, we performed this reconstruction for patients who were willing to have this operation.

There were 201 males and 203 females. Mean age was $69.0 \pm 12.9$ years. Preoperative echocardiography revealed an average peak pressure gradient through the aortic valve of $79.6 \pm 32.5 \mathrm{~mm} \mathrm{Hg}$ for patients with AS and a surgical annular diameter of $20.8 \pm 3.2 \mathrm{~mm}$ for all patients. Two hundred eighty-nine patients had AS and 115 patients had AR. One hundred two patients had bicuspid aortic valves, 13 patients had unicuspid valves, and 2 patients had quadricuspid valves. Annulo-aortic ectasia was recorded in 8 patients. The other 13 patients had IE.

\section{Surgical Technique}

The surgical technique for our original aortic valve reconstruction has been reported previously. ${ }^{13}$ Preparation of autologous pericardium was started by cleaning fat and other redundant tissue on the outer surface of pericardium with the Harmonic scalpel (Ethicon Endo-Surgery, Inc, Cincinnati, Ohio). Pericardium was excised at least $7 \times 8 \mathrm{~cm}$. The excised pericardium was then treated with $0.6 \%$ glutaraldehyde solution with a buffer for 10 minutes. The treated pericardium was rinsed 3 times using physiologic saline solution for 6 minutes.

Human pericardium is usually thin near the cranial end and thick caudally near the diaphragm. We use the thinner part of pericardium for small cusps to make the movement smoother, and the thicker part for large cusps to make them able to tolerate more stress (Figure 1). All aortic valve reconstructive procedures were performed during cardioplegic arrest on cardiopulmonary bypass.

Briefly, diseased cusps are excised meticulously. In case there is severe calcification along the aortic annulus, the Cavitron Ultrasonic Surgical Aspirator (SonoSurg, Olympus, Tokyo, Japan) is very helpful in removing calcium without damaging annular tissue. Then, the distance between each commissure is measured with an originally invented sizing apparatus. The intercommissural distance is measured using the original sizing apparatus by giving the appropriate tension, similar to reproducing the annulus during diastole. Correct measurement is vital to complete this operation. The new cusp with the size corresponding to the measured value is trimmed with an original template from glutaraldehyde-treated autologous pericardium. Last, the annular margin of the pericardial cusp is sutured with running 4-0 monofilament stitches to each annulus. The smooth (inner) surface of pericardium is placed on the left ventricular side. A running suture should be used to produce the natural, 3-dimensional bulge of the cusps effectively (Figure 2). The pericardial cusp is sewn thoroughly up to the top of commissure, and it is designed to have a deep coaptation that reaches up to the same horizontal plane as the commissure (Figure 3, $A$ ). The commissural coaptation is secured with additional 4-0 monofilament sutures. Recently, we added the small wing at the commissural part of pericardial cusps to get more tolerance to shear stress and even better coaptation (Figure $3, B$ and $C$ ). The coaptation of 3 new cusps is ensured with a visual check under negative pressure made by the left ventricular vent before closure of the aortotomy (Figure 3,D).

\section{Follow-up}

All the patients were monitored at the outpatient clinic of our hospital or at the referral hospital. Echocardiographic evaluation has been done 1 week, 1 month, 3 months, and every 6 months after surgery.

\section{Statistical Analysis}

Data are presented as mean \pm standard deviation. Survival rate and freedom from reoperation rate were calculated by Kaplan-Meier method.

\section{RESULTS}

No operation to convert to a prosthetic valve replacement was required. Aortic valve disease with IE and PVE could be operated in the same fashion. For AR with annuloaortic ectasia, our original aortic valve reconstruction could be combined effectively with the aortic root reimplantation technique. Mean aortic crossclamp time was $110.1 \pm 26.8$ minutes, and mean cardiopulmonary bypass time was $149.4 \pm 29.9$ minutes for isolated aortic valve reconstructions. Mean ischemic time was reduced to $96.7 \pm 26.9 \mathrm{~min}-$ utes among patients operated from January 2011 through September 2011. We have never resumed cardiopulmonary bypass to place additional sutures or to readjust the heights of the commissure or redundancy of the cusps.

Mean follow-up was $23.7 \pm 13.1$ months. Survival rate was $87.7 \%$ at 53 months (Figure $4, A$ ). The freedom from reoperation rate was $96.2 \%$ (Figure $4, B$ ). Reoperations were recorded for 2 patients, both because of IE. One was 10 months after the first operation and was induced by pacemaker implantation; the other was for a patient on hemodialysis 3.5 years after the first operation. These two

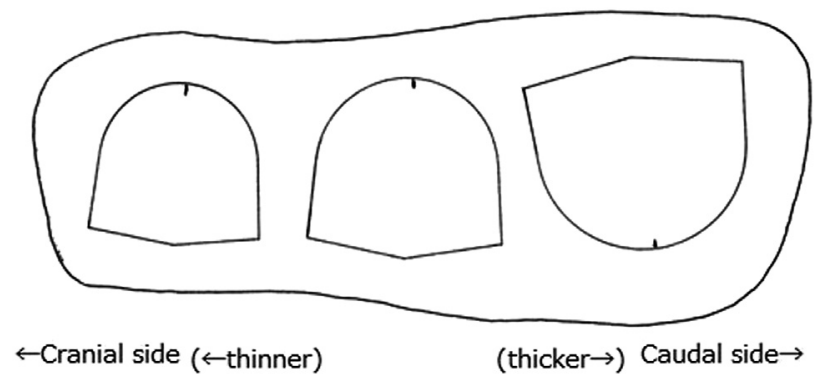

FIGURE 1. Management of pericardium. Generally speaking, pericardium becomes thicker as it approaches the diaphragm. On the other hand, it becomes thinner as it reaches the aorta. A larger cusp tends to have the more shear stress, and its opening/closing movement can be smooth regardless of its stiffness. Therefore, we always make the larger cusp from the diaphragm side and the smaller cusps from the aortic side. 




FIGURE 2. Suturing technique of the pericardial cusp. The suture is begun at the center of the annulus. This suture is tied and the ends are continued as a running suture bidirectionally. The ratio of distance between each bite on the pericardium and the annulus should be 3:1 at the bottom of the annulus, and it should be changed to $1: 1$ when the suture comes up to the coaptation zone.

reoperated patients were the only valve-related complications in this series. There were 7 in-hospital deaths with a noncardiac cause. The cause of in-hospital deaths included 3 cases of pneumonia, 2 cases of thrombotic thrombocytopenic purpura, 1 tracheal perforation, and 1 acute mesenteric ischemia. Twenty mortalities were recorded during the follow-up period after discharge from the hospital. No embolic event was recorded while patients received a small dose of daily aspirin. Basically, we do not provide anticoagulation postoperatively. Anticoagulation was used for 100 patients because of the concomitant procedure including coronary artery bypass surgery, mitral valve repair, and tricuspid valve repair.

Preoperative average peak pressure gradient through the aortic valve with echocardiography was $73.5 \pm 38.2 \mathrm{~mm}$ $\mathrm{Hg}$. The pressure gradient decreased $19.8 \pm 3.7 \mathrm{~mm} \mathrm{Hg} 1$ week after surgery and $13.8 \pm 3.7 \mathrm{~mm} \mathrm{Hg} 3.5$ years after surgery (Figure 5, $A$ ). The degree of aortic regurgitation with echocardiography was less than mild postoperatively (Figure 5, B).

\section{DISCUSSION}

Aortic valve repair became feasible with low mortality in many patients with aortic regurgitation. It is showing better results in terms of freedom from valve-related complications compared with conventional aortic valve replacement. ${ }^{14}$ The important milestones in the development of aortic valve repair have already been achieved as it evolves into a viable alternative to aortic valve replacement in patients with aortic regurgitation. ${ }^{15}$ The origin of aortic valve repair can be traced back to the early era of cardiac surgery. ${ }^{16}$ Aortic valve repair has been performed using native valve cusps by various techniques that include commissurotomy, ${ }^{17}$ annuloplasty, free edge unrolling, cusp resuspension, supra-aortic crest enhancement, free edge reinforcement, wedge resection, and so on. ${ }^{18}$ This type of conservative repair is not always possible, especially for calcified AS in aging elderly patients. Simple decalcification or slicing of cusps has not shown good results. On the other hand, there is an apparent limitation of durability with bioprosthetic valves and an apparent disadvantage of anticoagulation with mechanical prostheses. Moreover, both prostheses cannot create good hemodynamics compared with a native aortic valve.

Attempts to replace aortic valve cusp tissue with biologic material have been made since the late 1960s. ${ }^{19-21}$ Fascia lata, dura mater, and bovine pericardium have been used in a small number of patients, but the results have not been favorable in the majority of instances. Halees and colleagues ${ }^{4}$ reported that autologous pericardium and bovine pericardium did not show a difference in terms of structural valve deterioration with 16 years of follow-up of aortic valve reconstruction. But, they found more cases of late calcification with bovine pericardium than with autologous pericardium. ${ }^{4}$ Autologous pericardium is easily available for immediate aortic valve repair in every patient.

Aortic valve repair using autologous pericardial cusp extension has been described by Duran and associates. ${ }^{9,18,22,23}$ They treated autologous pericardium with glutaraldehyde solution. The treated pericardium was used to increase the height of native aortic valve cusps and commissures, resulting in an increase of the coaptation zone. Glutaraldehyde treatment can provide more resistance against retraction and degeneration, and maintains intrinsic tissue pliability to the pericardium. We also used $0.6 \%$ glutaraldehyde solution with buffer to treat pericardium. The ultimate tensile strength of glutaraldehyde-treated human pericardium was 4 times greater than noncalcified leaflets, indicating its suitability for application to aortic valve reconstruction. ${ }^{24}$

Halees and colleagues ${ }^{4}$ reported an overall reoperationfree survival of $47 \%$ at 16 years after aortic valve reconstruction with pericardium. It is their contention that cusp extension or replacement of the 3 aortic cusps with a single strip of pericardium is technically more reliable. Odim and coworkers ${ }^{25}$ reported that aortic valve repair with autologous pericardial cusp extension was a good alternative for a congenitally diseased aortic valve. Chan and colleauges ${ }^{5}$ reported 11 patients of truly stentless autologous pericardial aortic valve replacement. With their 6.5 years of follow-up, freedom from structural valve deterioration was $100 \%$. One patient was reoperated 15 months after surgery, and the replaced autologous pericardium showed no calcification and had well-preserved collagen fibers. 

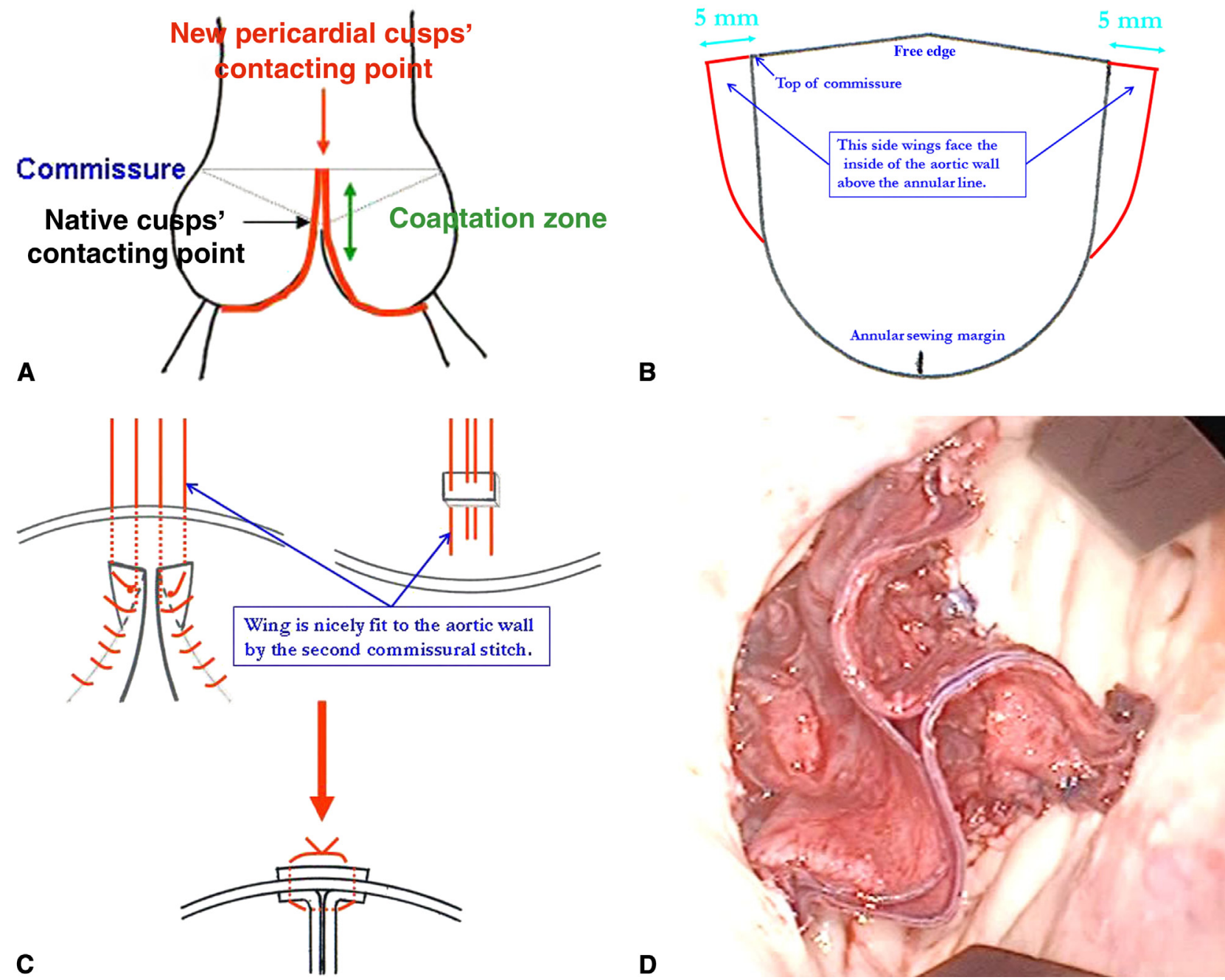

C

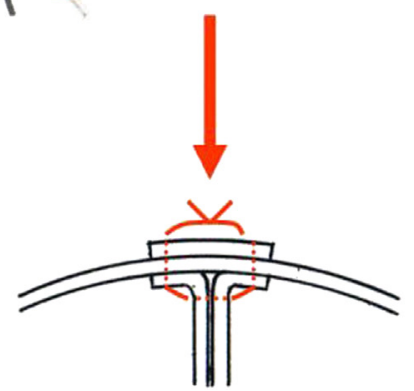

D

FIGURE 3. A, Concept of commissure height. An original template was designed to make pericardial leaflets have deep coaptation up to the same horizontal plane as the top of the commissures. B, Commissure creation. With the commissural wing sewed facing the aortic wall, the commissural part of each cusp can have more strength to tolerate the maximal stress, and the commissural part of each cusp bulges naturally toward the aortic lumen, which gives better coaptation for the cusps. C, upper left, The first annular suture is pulled out around the center of wing along the annular suture marking line to just inside (below) the native commissure. Second commissuroplasty suture (4-0 prolene RB-1) is first passed through both sides of cusps just above the last bite of annular stitch along the annular suture marking line. Then, the second commissuroplasty suture is passed through more laterally and upward near the edge of wing, and through the aortic wall above the commissure to fit this wing against the aortic wall. Upper right, Annular stitches travel more narrow and lower (inside the commissure), and commissuroplasty stitches travel wider and higher (outside the commissure). This is same on the felt pledget. Lower, The wings should fit nicely to the aortic wall above the annular line. D, Real completion of aortic valve reconstruction including commissuroplasty.

Our original aortic valve reconstruction technique does not focus on total repair with a single strip of pericardium. On the contrary, we make much of the way of thinking that the aortic valve represents a collection of different-size cusps, so we consider the size of the aortic cusp to be defined independently by the distance between the commissure. As a result, we create pericardial cusps separately for each coronary cusp, and each cusp may be a different size from the other. Based on our collected data, aortic valves represent a collection of different-size cusps.

Among tricuspid patients, $13 \%$ showed 3 equidistant measurements between commissures. Fifty-seven percent of patients had 2 equidistant measurements, and the remaining 30\% had 3 different distances.

With native aortic valves, the cusp contacting point is slightly lower compared with the horizontal plane made by the top of the commissures (Figure 3, A). Its level is different in each case. If the cusp contact point is far below the commissural level, the cusp free-edge length is made longer. If the cusp contact point is near the commissural level, the cusp free-edge length is made shorter. We thought it difficult to calculate the cusp free-edge length from the annular diameter in consideration of these factors. 

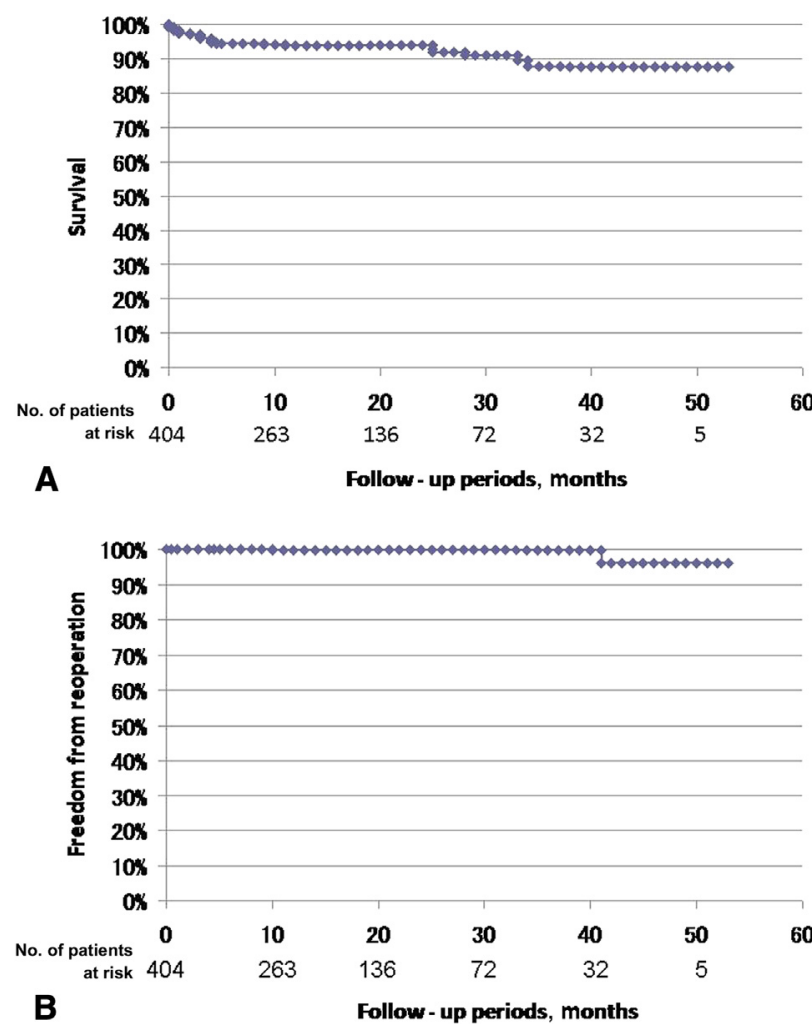

FIGURE 4. A, Survival curve with $95 \%$ confidence limits. B, Freedom from reoperation curve with $95 \%$ confidence limits.

There are 2 essential features of our original reconstruction that is completely different from others. First, we calculate cusp free-edge length from the intercommissural length and second, the contact point of the new pericardial cusps is raised up to the same plane of the commissures. From these two concepts, we have realized two advantages. First, we are able to have a larger coaptation zone and, second, we can determine the cusp free-edge length from the distance between the commissure on the same 2-dimensional plane as one-to-one correspondence. Therefore, it becomes easy to recognize the area of each cusp (Figure 3,D). Our concept is based on the premise that the length of each cusp free edge is in proportion to the distance between each commissure. This value is sufficient to determine the new size of cusps, rather than fully calculating each cusp area. With our template, the size of the new pericardial cusp is determined by 3 factors: the length of the cusp free edge, the larger coaptation zone, and the margin for seams. Correct measurement of the distance between each commissure and a suturing technique with a ratio of 3:1 at the bottom and 1:1 the near commissures to produce cusps that have a nice round bulge are the keys to this procedure. If these 2 factors are done well, reconstruction should be successful. In fact, we have never resumed cardiopulmonary bypass to place additional sutures or to readjust the heights of the commissure or redundancy of the cusps.

We believe independent replacement of cusps is more effective in preserving the natural motion of the aortic valve annulus and the coordination of the left ventricle, aortic annulus, sinus of Valsalva, and aorta. With our technique of total resection of all 3 diseased cusps, our procedure can be applied to any type of aortic valve disease. In fact, we can operate on patients with AS, AR, IE, and PVE, and those with bicuspid, unicuspid, ${ }^{26}$ and quadricuspid ${ }^{27}$ valves, basically in the same fashion. Even for annulo-aortic ectasia, this technique can be applied with root reimplantation. This wide applicability can be attributed also to the independent reconstruction of 3 cusps. In a situation with an intact aortic annulus, this new reconstruction technique can be applied to all kinds of aortic valve diseases. To secure the intact aortic annulus, the Cavitron Ultrasonic Surgical Aspirator is used to remove calcium without damaging annular tissue.

\section{Study Limitations}

The limitations of this study relate to its retrospective nature and the limited follow-up. Moreover, this study was not a case-control study. A prospective case-control study would be ideal to determine the efficacy of our new aortic valve reconstruction compared with other conventional aortic valve replacement or repair procedures. Long-term results have to be studied with a follow-up of 15 to 20 years.

Although our cardiopulmonary bypass time and aortic crossclamp time were longer than seen in standard aortic valve
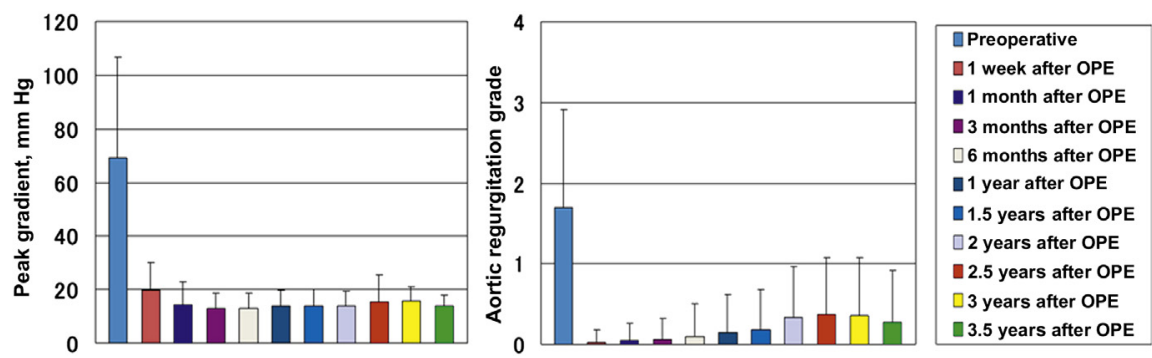

FIGURE 5. Left, Follow-up echocardiographic evaluation of peak pressure gradient after aortic valve reconstruction. Right, Follow-up echocardiographic evaluation of aortic regurgitation after aortic valve reconstruction. 
replacement, we were able to provide a better patient quality of life, with good hemodynamics and a warfarin-free condition. And, with good hemodynamics and a natural, smooth motion of pericardial cusps, we can expect better longevity compared with previously reported techniques or materials.

\section{CONCLUSIONS}

Our original aortic valve reconstruction provides good short- and mid-term results along with good hemodynamics and a good quality of life without anticoagulation. This procedure is effective for a wide variety of aortic valve diseases. Assessment of long-term data, especially concerning valve durability, will be disclosed in the future.

We thank Prof Hartzell V. Schaff for giving us suggestions to complete this manuscript.

\section{References}

1. Ozaki S. Pathophysiology of calcification of bioprosthetic heart valves: an experimental investigation. Acta Biomedica Lovaniensia. Vol. 238. Leuven University Press; 2001

2. Lansac E, Di Centa I, Raoux F, Al Attar N, Acar C, Joudinaud T, et al. A lesional classification to standardize surgical management of aortic insufficiency towards valve repair. Eur J Cardiothorac Surg. 2008;33:872-80.

3. Doss M, Sirat S, Risteski P, Martens S, Moritz A. Pericardial patch augmentation for repair of incompetent bicuspid aortic valves at midterm. Eur J Cardiothorac Surg. 2008;33:881-4.

4. Halees ZA, Shahid MA, Sanei AA, Sallehuddin A, Duran C. Up to 16 years follow-up of aortic reconstruction with pericardium: a stentless readily available cheap valve? Eur J Cardiothorac Surg. 2005;28:200-5.

5. Chan KMJ, Rahman-Haley S, Mittal TK, Gavino JA, Dreyfus GD. Truly stentless autologous pericardial aortic valve replacement: an alternative to standard aortic valve replacement. J Thorac Cardiovasc Surg. 2011;141:276-83.

6. Aupart MR, Sirinelli AL, Diemont FF, Meurisse YA, Dreyfus XB, Marchand MA. The last generation of pericardial valves in the aortic position: ten year follow-up in 589 patients. Ann Thorac Surg. 1996;61:615-20.

7. Banbury MK, Cosgrove DM, Lytle BW, Smedira NG, Sabik JF, Saunders CR. Long-term results of the Carpentier-Edwards pericardial aortic valve: a 12 year follow-up. Ann Thorac Surg. 1998;66:S73-6.

8. Grabenwoger M, Fitzal F, Gross C, Hutschala D, Bock P, Brucke P, et al. Different modes of degeneration in autologous and heterologous heart valve prostheses. J Heart Valve Dis. 2000;9:104-9.
9. Duran CMG, Gometza B, Kumar N, Gallo R, Martin-Duran R. Aortic valve replacement with freehand autologous pericardium. J Thorac Cardiovasc Surg. 1995;110:511-6.

10. Cheng A, Dagum P, Miller DG. Aortic root dynamics and surgery: from craft to science. Philos Trans R Soc Lond B Biol Sci. 2007;362:1407-19.

11. Rodriguez F, Green G, Dagum P, Nistral JF, Harrington KB, Daughters GT, et al. Left ventricular volume shifts and aortic root expansion during isovolumic contraction. J Heart Valve Dis. 2006;15:465-73.

12. Lansac E, Lim HS, Shomura Y, Lim KH, Rice NT, Goetz W, et al. A four-dimensional study of aortic root dynamics. Eur J Cardiothorac Surg. 2002;22:497-503.

13. Ozaki S, Kawase I, Yamashita H, Uchida S, Nozawa Y, Matsuyama T, et al. Aortic valve reconstruction using self-developed aortic valve plasty system in aortic valve disease. Interact Cardiovasc Thorac Surg. 2011;12:550-3.

14. Aicher D, Fries R, Rodionycheva S, Schmidt K, Langer F, Schafers HJ. Aortic valve repair leads to a low incidence of valve-related complications. Eur J Cardiothorac Surg. 2010;37:127-32.

15. Boodhwani M, El Khoury G. Aortic valve repair: a glimpse into the future. Eur J Cardiothorac Surg. 2012;41:2-3.

16. Lillehei CW, Gott VL, De Wall RA, Varco RL. The surgical treatment of stenotic or regurgitant lesions of the mitral and aortic valves by direct vision utilizing a pump oxygenator. J Thorac Cardiovasc Surg. 1958;35:154-91.

17. Hill DG. Long term results of debridement valvotomy for calcific aortic stenosis. J Thorac Cardiovasc Surg. 1973;65:708-11.

18. Duran CMG, Alonso J, Gaite L, Alonso C, Cagigas JC, Marce L, et al. Long-term results of conservative repair of rheumatic aortic valve insufficiency. Eur J Cardiothorac Surg. 1988;2:217-23.

19. Bjork VO, Hultquist G. Teflon and pericardial aortic valve prostheses. J Thorac Cardiovasc Surg. 1964;47:693-701.

20. Ross DN. Aortic valve disease and left ventricular obstruction. In: Cleland W, Goodwin J, McDonald L, Ross DN, eds. Medical and surgical cardiology. Oxford: Blackwell; 1969:997.

21. Senning A. Fascia lata replacement of aortic valves. J Thorac Cardiovasc Surg. 1967;54:465-70.

22. Duran CM, Gometza B, Kuma N, Gallo R, Bjonastad K. From aortic cusp extension to valve replacement with stentless pericardium. Ann Thorac Surg. 1995;60:S428-32.

23. Duran C, Gometza B, Kuma N, Gallo R, Bjonastad K. Treated bovine and autologous pericardium: surgical technique. J Cardiac Surg. 1995;10:1-9.

24. Yamashita H, Ozaki S, Iwasaki K, Kawase I, Nozawa Y, Umezu M. Tensile strength of human pericardium treated with glutaraldehyde. Ann Thorac Cardiovasc Surg. 2012;18:434-7.

25. Odim J, Laks H, Allada Y, Child J, Wilson S, Gjertson D. Results of aortic valve sparing and restoration with autologous pericardial leaflet extension in congenital heart disease. Ann Thorac Surg. 2005;74:438-43.

26. Kawase I, Ozaki S, Yamashita H, Uchida S, Nozawa Y, Matsuyama T, et al. Aortic valve reconstruction of unicuspid aortic valve by tricuspidization using autologous pericardium. Ann Thorac Surg. 2012;94:1180-4.

27. Kawase I, Ozaki S, Yamashita H, Uchida S, Nozawa Y, Matsuyama T, et al. Original aortic valve plasty with autologous pericardium for quadricuspid valve. Ann Thorac Surg. 2011;91:1598-9. 